\title{
La oclusión como factor etiopatológico en los trastornos temporomandibulares
}

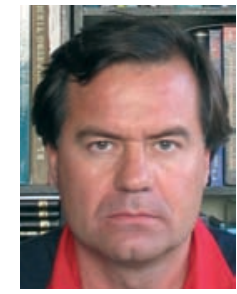

García-Fajardo Palacios, Carlos

\author{
The occlusion as an etiopathological risk factor in remporomandibular disturbance
}

\section{García-Fajardo Palacios, Carlos* Cacho Casado, Alberto Fonte Trigo, Abelardo*** Pérez -Varela, Juan Carlos***}

*Médico Odontólogo, Postgrado en Diagnóstico y Patología de la ATM y Dolor Orofacial y miembro de la American Academy of Craniofacial Pain. Autor del libro "Dolor odontoestomatológico»

**Doctor en Medicina y Cirugía UCM. Profesor titular en la Universidad Complutense de Madrid.

$* * *$ Ortodoncista exclusivo. Master ortodoncia y ortopedia dentofacial Universidad Internacional Cataluña

****Profesor de Ortodoncia y Ortopedia en la Universidad de Santiago de Compostela. Miembro diplomado de la SEDO.

\section{Correspondencia}

cf@carlosgarciafajardo.com
Resumen: Fundamento. La relación intrínseca entre alteración oclusal y trastorno articular no es un paradigma que se cumpla siempre. Sin embargo, el análisis oclusal debe ser siempre un requisito imprescindible en la exploración del paciente disfuncional ya que multitud de estudios demuestran que puede representar un factor etiológico de primer orden. Material y método. A través de distintas revisiones bibliográficas se describen los principales estados oclusales y se intenta establecer una posible relación estructural y funcional entre los distintos cuadros articulares. Resultados. La relación entre cada tipo de maloclusión y un determinado trastorno temporomandibular (TTM), se evidencia a través de las distintas posiciones condilares en la cavidad glenoidea. Se podría hablar de una Unidad DienteCóndilo ya que el aparato estomatognático posee un singular bloque óseo, como es la mandíbula, que alberga en su misma estructura a dientes y cóndilo, lo que implicaría que las distintas variantes de la oclusión se trasladen de manera dinámica a la articulación. Conclusión. Aunque la implicación del factor oclusal en la etiopatología temporomandibular está condicionada por la presencia de otros factores coadyudantes, el reestablecimiento de la oclusión fisiológica puede ser primordial para reestablecer la homeostasis articular e implicaría conocer la biomecánica particular de cada paciente y su correlación con sus parámetros biológicos.

Palabras clave: Disarmonia oclusal. Interferencia oclusal. Articulación temporomandibular. Cóndilo. Maloclusion en la disfunción temporomandibular.

Abstract: Background: The relationship between occlusal discrepancies and TMJ disorders does not always hold true. Nonetheless, occlusal analysis is a requisite to achieve a correct diagnosis in the dysfunctional patient. Many studies show that occlusal discrepancies can be a very important etiological factor. Material and Methods: Various literature reviews describe the main occlusal types and the possibility of structural and functional relationships with TMJ derangements. Results: The relationship between each kind of malocclusion and a specific TMJ disorder is due to different condyle positions in the glenoid fossa. It may be appropriate to introduce the term Tooth-Condyle Unit because the stomatognathic system has a specific bone block or mandible, which encompass teeth and condyles. The various occlusal schemes can be considered dynamic which influences the joint. Conclusion: The involvement of occlusion in the etiology of TMJ disorder is conditioned by the presence of other factors. However, physiologic occlusion must be re-established for correct joint function. This implies that the specific biomechanics of each patient and it's correlation with biological parameters must be known in order to provide joint homeostasis..

Key words: Occlusal disharmony. Occlusal interferences. Temporomandibular joint. Condyle. Malocclusion in TMJ dysfunction.

BIBLID [1138-123X (2007)12:1-2; enero-junio 1-104]

García-Fajardo Palacios C, Cacho Casado A, Fonte Trigo A, Pérez-Varela JC.La oclusión como factor etiopatológico en los trastornos temporomandibulares. RCOE 2007;12(1-2):37-47. 


\section{Etiopatología de los trastornos temporomandiulates [TM]}

Según Wagner de Oliveira, la etiología de la disfunción temporomandibular es uno de los asuntos más controvertidos y estudiados de la Odontología. Quizá porque no se encuentra una clara relación causa-efecto o porque faltan fundamentos científicos concretos pero, sin embargo, sí parece haber consenso en la comunidad científica de que se trata de un trastorno multifactorial que se desglosa en factores predisponentes que aumentan el riesgo de la aparición de un trastorno, factores desencadenantes que provocan en última instancia el comienzo de un trastorno y los factores perpetuantes que impiden la curación y favorecen el agravamiento'.

Distintos factores podrían pertenecer a más de uno de estos efectos, e incluso un sólo factor podría tener uno o más de esos efectos. En el siguiente cuadro (Tabla 1) se agrupan de forma indiscriminada los principales factores que pueden intervenir en la aparición de un trastorno temporomandibular, ya sea predisponente, perpetuante o desencadenante.

Este cuadro no representa la frecuencia estadística ni la importancia de un factor $u$ otro en la incidencia de los TTM, sino que muestra gráficamente que todos los factores pueden ser importantes y ninguno es imprescindible y que cuantos más concurran

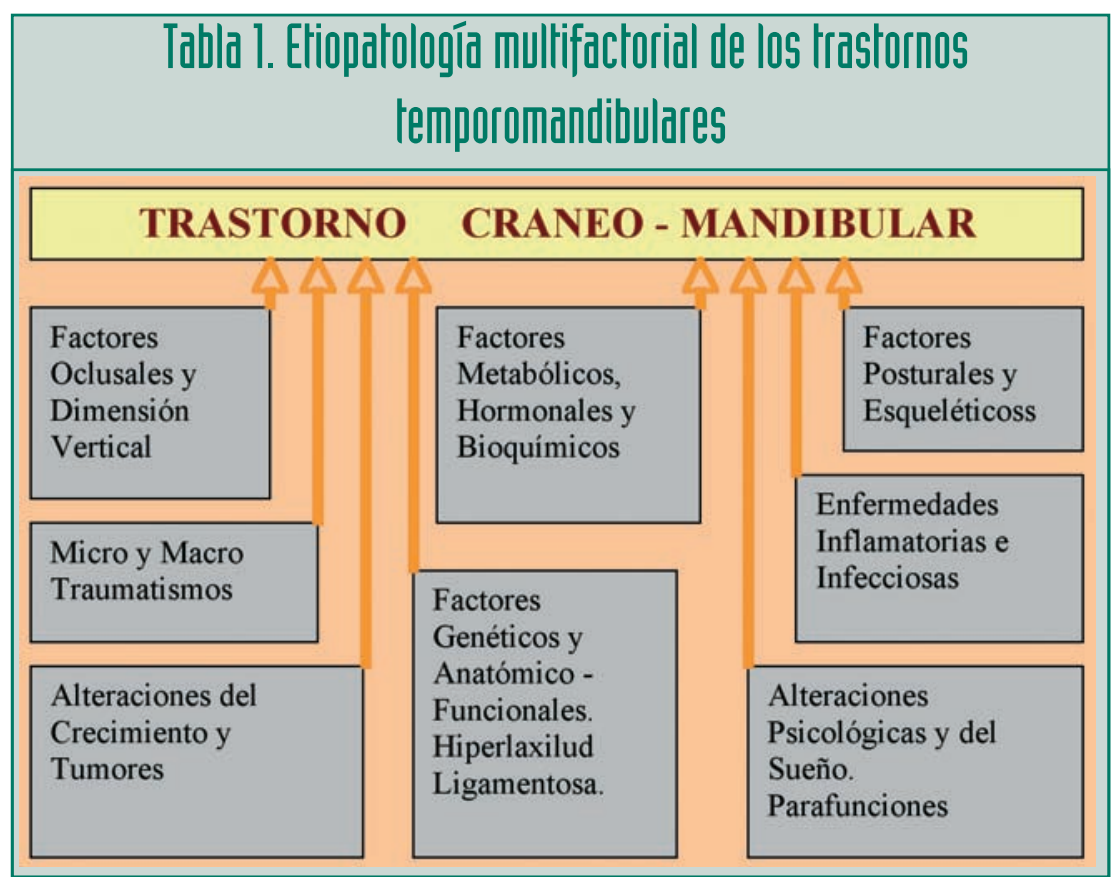

en el tiempo y el espacio más probabilidades habrá de que aparezca un TTM.

No hay que olvidar tampoco la tolerancia fisiológica y funcional propia de cada paciente y que va a condicionar la variabilidad en la aparición y Iocalización del problema. Cada estructura individual del sistema masticatorio posee un grado de tolerancia a la agresión. Cuando éste supera un valor crítico se desencadena el trastorno. Es decir, que la disfunción no se manifiesta en todas las personas por igual, sino que se refleja precisamente en esa estructura más débil que cada paciente tiene (menor tolerancia estructural) y que hace que la variabilidad sea aún más marcada entre unos individuos y otros.

\section{Historia de la oclusión como causa de los TTM}

En los tratamientos ortodóncicos y con el desarrollo de nuevas técnicas de diagnóstico, los objetivos terapéuticos se ampliaron para conseguir la estabilidad de los resultados. Sin embargo, a pesar de esta evolución, se empezó a observar que tratamientos con resultados óptimos presentaban problemas de estabilidad, abrasiones dentarias, movilidad y lo más importante, problemas en la articulación temporomandibular.

Para solventar este problema, el diagnóstico de la anomalía debe considerar la posición de las bases esqueléticas en una correcta relación céntrica, teniendo en cuenta la influencia que sobre los dientes ejercen los músculos y ligamentos circundantes. La literatura respecto a 
este campo es extensa y, por tanto, un tanto contradictoria en ciertos puntos, aunque la mayoría apuntan a una relación entre la presencia de una maloclusión y la aparición de la disfunción craneomandibular (DTM). Fue Costen en 1934 quién describe un complejo de síntomas que incluyen la pérdida del soporte oclusal dentario acompañado de síntomas de audición como dolor y tínitus o dolor en el seno. Desde entonces, otros investigadores han demostrado Ios efectos beneficiosos del ajuste oclusal para eliminar esta sintomatología². En 1980, Weinberg y Linn comprobraron que una modificación súbita en la oclusión puede causar dolor craneomandibular agudo.

Por otra parte, se ha encontrado un aumento, pequeño pero significativo $(+5 \%)$, del porcentaje de mordidas abiertas anteriores y mordidas cruzadas posteriores en los pacientes con DTM. De hecho, parece que estos individuos con problemas verticales y transversales desde edades tempranas podrían ser más propicios a desarrollar patologías articulares. En este sentido, Pullinger y colaboradores, en 1993, relacionaron maloclusiones como la mordida abierta anterior, la mordida cruzada unilateral, un resalte mayor de $6 \mathrm{~mm}$, una pérdida de cinco o más dientes posteriores y la discrepancia entre RC y OC mayor de 5 $\mathrm{mm}$, con un mayor riesgo de padecer DTM. Otras maloclusiones dentoesqueléticas y dentales como las Clases II, ya sean división I o II, y las Clases III han sido asociadas a trastornos articulares por Riolo en 1987, Al Hadi en 1993 y otros muchos autores.

En lo que se refiere al tratamiento con ortodoncia de estos desajustes

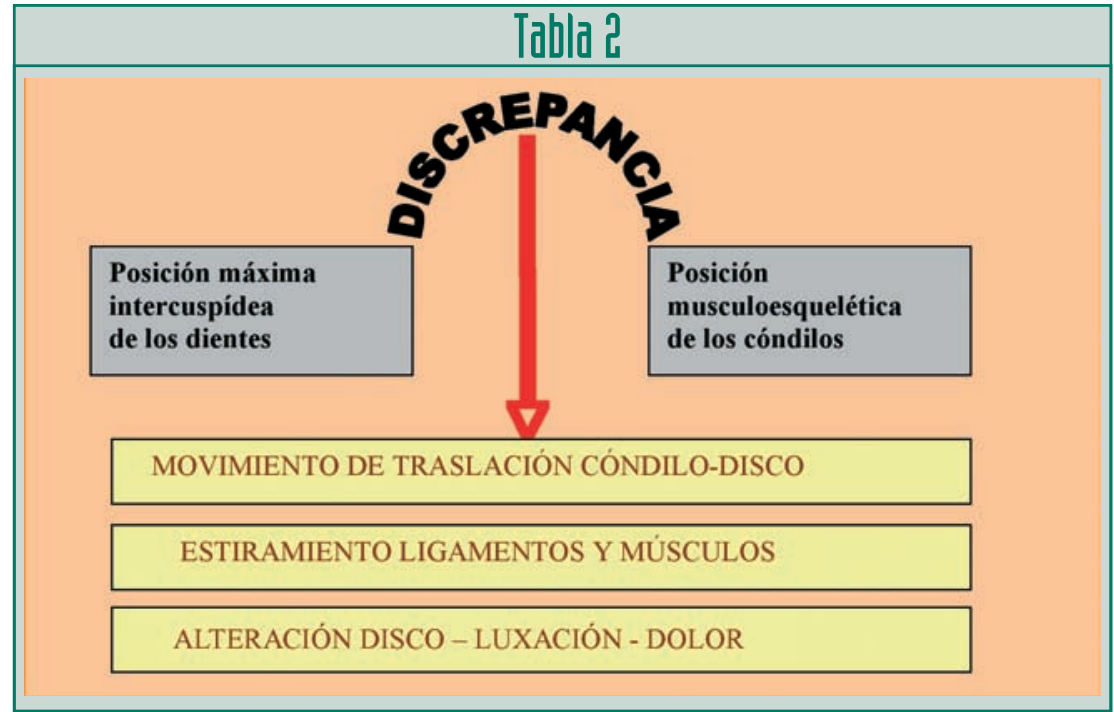

oclusales, no representa un factor de riesgo en el desarrollo de la DTM, pero si se ha observado que los individuos que recibieron tratamientos de ortodoncia en la adolescencia presentan menos tendencia a padecer trastornos relacionados con la ATM en la edad adulta como refieren Egermark y Thilander en 1992.

No debemos dejar de mencionar en esta revisión un factor tan importante como es el estrés. Autores como Moody en 1982 nos mostraron que los pacientes con DTM mostraban niveles de unidades estresantes mayores que los pacientes con otras dolencias. Stein en 1982 y Fearon y Serwatka un año más tarde afirmaban que el nivel de estrés de pacientes con DTM era significativamente mayor que en el grupo de pacientes control. En la misma dirección, Niemi y Le Bell, en 1993, mostraron que los pacientes con DTM presentaban mayores niveles de estrés. Si profundizamos más en este sentido podemos llegar a encontrar relaciones establecidas entre la DTM y ansiedad, sentimientos de cul- pa, tristeza, hipocondría e incluso otros trastornos neurodepresivos severos descritos por autores como Schnurr en 1990, Parker en 1993, Curran en 1996 o Ruf en 1997, entre otros.

Nosotros consideramos que el papel de la oclusión debe adquirir el grado justo de participación en la etiopatogénesis de los TTM. Y éste no es otro que el determinado por el estudio pormenorizado de todos los factores posibles en función de los parámetros individuales de cada paciente.

En este estudio que presentamos se describen las posibles alteraciones a nivel temporomandibular que pueden concurrir a partir de un estado oclusal alterado. Posiblemente, la única presencia de un estado oclusal alterado nunca será suficiente para desencadenar un TTM, si bien la asociación con otros factores de riesgo (como los expuestos anteriormente y en la tabla I) podrían desencadenar o perpetuar el trastorno. Por lo tanto se debe siempre valorar el estado oclusal 
como un peldaño más en la búsqueda del factor o los factores etiopatológicos diferenciales.

\section{Relaciones estáticas 4 dinámicas}

Una de las razones por las cuales es posible que los estudios sobre la oclusión sean tan variables es por considerar la oclusión desde un punto de vista estático. La estabilidad ortopédica existe cuando la posición intercuspídea estable de los dientes está en armonía con la posición musculoesquelética estable de los cóndilos en las fosas articulares (Fig.14).

Cuando existe una discrepancia entre estas dos posiciones, uno de los cóndilos o ambos no se sitúa en una posición estable con el disco y la fosa (tabla 2) y aumenta el riesgo de alteraciones intracapsulares ${ }^{3}$.

Existen dos factores que marcan la gravedad y repercusión clínica de ésta inestabilidad ortopédica:

1) EL GRADO DE INESTABILIDAD. A partir de 2-3 mm, el riesgo de alteraciones intracapsulares aumenta.

2) LA MAGNITUD DE LA CARGA. Si el paciente asocia la inestabilidad con episodios de bruxismo o cualquier parafunción que aumente la carga, el riesgo aumentará también.

Por eso, es posible encontrarse casos con una gran maloclusión con mordida abierta, por ejemplo, pero sin inestabilidad ortopédica o sin bruxismo y por lo tanto el paciente puede vivir sin sintomatología dolorosa ni alteraciones intracapsulares. Sin embargo, otro paciente con una oclusión aparente más correcta, pero que pre- senta una gran inestabilidad ortopédica y bruxismo, podría padecer dolor y disfunción temporomandibular.

Todos estos parámetros son sin duda el caballo de batalla en la atribución del papel de la oclusión y su grado de implicación en la etiopatología de los trastornos temporomandibulares que han suscitado, en las distintas escuelas, no pocas polémicas y controversias.

\section{Estudio de la posible} repercusión del estado oclusal en la homeosta-
sia temporomandibular

La oclusión dental está determinada por el crecimiento óseo, el desarrollo de la dentición y la madurez neuromuscular y representa un posible factor etiológico en patología disfuncional, sobretodo si se asocia al stress.

Existen multitud de estudios para clasificar las distintas alteraciones y disarmonías oclusales en base a diferentes parámetros. En la tabla 3 hemos elaborado una síntesis de los distintos estados que pueden interferir en el correcto funcionamiento temporomandibular en base a los tres grandes grupos que engloban la patología oclusal disfuncional:

\section{Clase II, 1}

Son pacientes (fig.1) que en los movimientos protrusivos no presentan una disoclusión inmediata sobre los dientes anteriores debido al característico Resalte. Como resultado

\begin{tabular}{|l|}
\hline Tabla ? \\
\hline Maloclusiones \\
a) Clase II/1 \\
b) Clase II/2 \\
c) Clase III \\
d) Mordida abierta anterior \\
e) Mordida cruzada \\
Interferencias \\
a) Interferencia en protrusiva \\
b) Interferencia en trabajo \\
c) Interferencia en balanceo \\
d) Interferencia en oclusión cén- \\
trica \\
e) Discrepancia Oc-RC \\
Alteraciones funcionales \\
y de la dimensión vertical \\
a) Disminución de la dimensión \\
b) Aumento de la dimensión \\
c) Alteraciones funcionales y de \\
la masticación \\
\hline
\end{tabular}

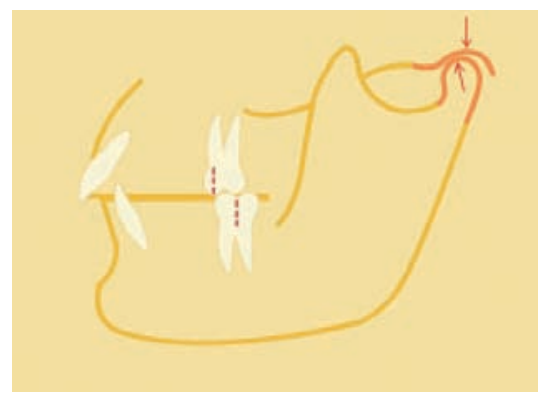

Figura 1

inmediato podría haber interferencias oclusales posteriores que transmitan las fuerzas de oclusión al periodonto ${ }^{4}$ y a la ATM, así como causar disfunción muscular. De cualquier forma, lo que sí sucede siempre es que hay un excesivo rango de función mandibular para llegar a producirse la disoclusión anterior derivado de la posición dental anterior.

Existen algunos pacientes con este tipo de oclusión (fig.2) que proyectan 


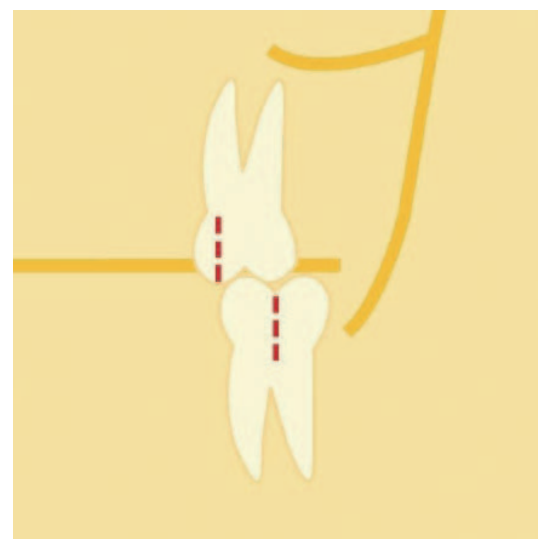

Figura 2

la mandíbula hacia delante (fig.3). Ello conlleva, logicamente, un estiramiento de músculos y ligamentos y en ocasiones a una compresión de los tejidos blandos adyacentes al disco articular. El sobreesfuerzo y fatiga debido a la contracción prolongada para mantener esta posición es claramente perjudicial. En literatura anglosajona se les ha llamado los Sunday bite porque en algunos casos y por razones estéticas evitan el aspecto facial de una mandíbula retruida proyectando ésta hacia delante.

\section{Clase II, 2}

En esta situación, al contrario de la anterior, el rango de movimiento está limitado debido a la excesiva sobremordida vertical y el efecto traumático de esta maloclusión se relaciona con una probable posición de los cóndilos desplazados e intruídos en la fosa (fig.4). Un desplazamiento más distal a este punto se encontraría con el espacio retrodiscal ricamente inervado y fuente del típico dolor temporomandibular retrodiscal ${ }^{5}$. Esta posición distal condilar extrema sería causa de inflamación de los tejidos y debilidad funcional de la articulación

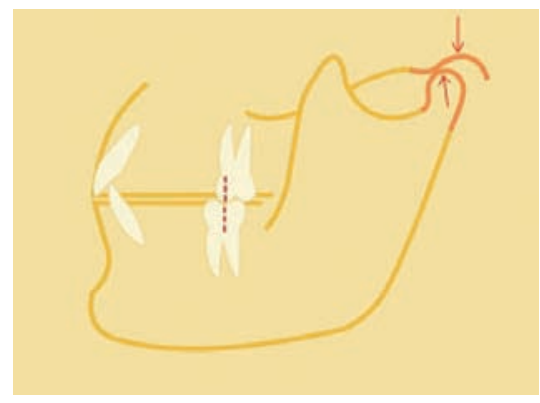

Figura 3

por estiramiento de los tejidos blandos y mayor pérdida de contacto discal.

Hay una guía anterior exagerada, de tal manera que no guarda sincronía la disoclusión anterior con la guía condilar ya que tienen distintos desplazamientos angulares. Es importante señalar que la salud articular y periodontal queda salvaguardada si existe una similitud entre la amplitud y arco del movimiento del cóndilo dentro de la cavidad y el movimiento y arco de los distintos movimientos de desoclusión. Un individuo con unas caras oclusales muy planas y sin guía anterior se correspondería con una articulación con una eminencia y una cavidad poco pronunciada y de igual manera ocurriría a la inversa.

En caso de pacientes con una clase II con gran sobremordida y un periodonto frágil, puede desembocar en fuerzas ejercidas sobre los dientes anteriores de manera traumática y prolongada provocando un microtrauma periodontal hasta desencadenar una migración en abanico exterior o splaying anterior (fig.5) con aparición de diastemas ${ }^{6}$. Sin embargo, si el periodonto presenta una gran fortale-

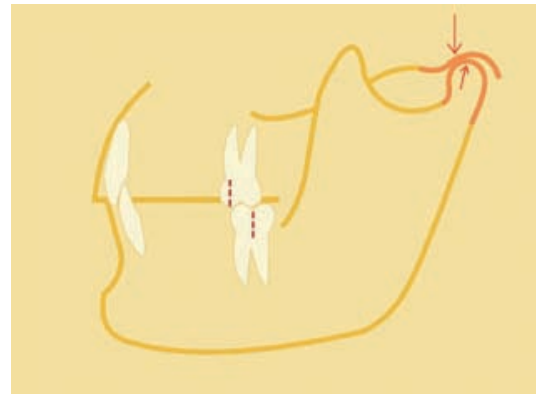

Figura 4

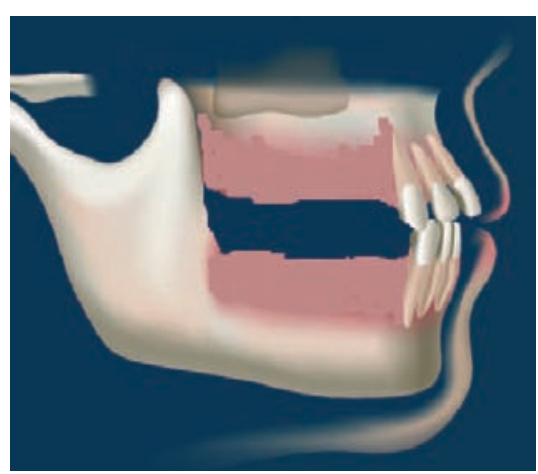

Figura 5

za ósea y no permite el desplazamiento y la migración dental, es la articulación temporomandibular la que puede sufrir un grado de stress mayor debido a esta gran sobremordida asociada.

\section{Clase III}

Estos pacientes pueden carecer de guía anterior (fig.6), y existir una falta de disoclusión anterior durante los movimientos protrusivos. Si se acompaña de una ausencia de guía canina, el contacto en las lateralidades tendrá que confiar en una función de grupo pero en caso contrario la presencia de interferencias en balanceo en los movimientos de lateralidad sería inevitable. Esta anomalía en los movi- 


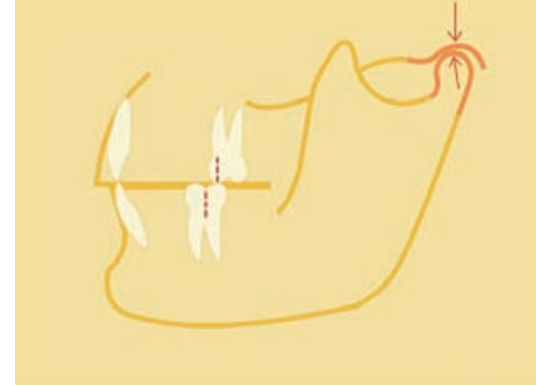

Figura 6

mientos excéntricos podría desencadenar, como se estudiará más adelante, en un traumatismo oclusal ó una disfunción temporomandibular?

\section{Mordida abierta anterior}

Usualmente, la causa primaria de la mordida abierta es la interposición lingual y el patrón de deglución atípica que empuja los dientes o impide su erupción completa. Puede ser simplemente de origen dental o estar acompañada de una deformidad ósea, aunque, en ambos casos, la ausencia de guía canina y la presencia de interferencias posteriores están siempre presentes. Se estudiará con más detalle en el apartado de interferencias.

\section{Mordida cruzada}

Aparentemente una mordida cruzada anterior (fig.7) podría simular una clase III esquelética pero un análisis ulterior de la oclusión céntrica puede demostrar que se trata de una mordida cruzada dental en clase I donde el cóndilo podría apreciarse, radiograficamente, en una posición más avanzada de tal forma que si lo situamos en su posición exacta en la fosa en relación céntrica podrían surgir fuertes interferencias posteriores que el paciente va a evitar colocando la mandíbula hacia delante en oclu-

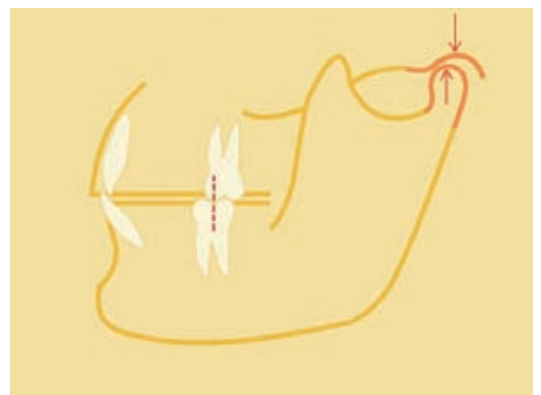

Figura 7

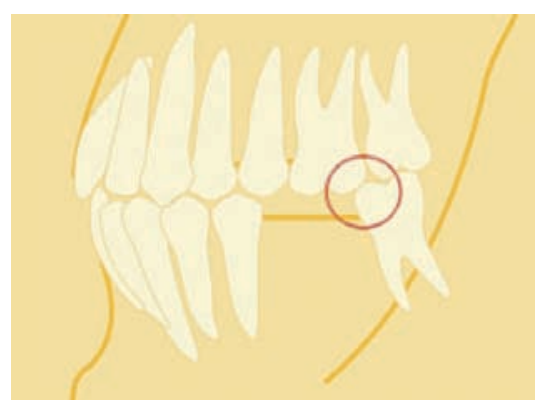

Figura 8 sión céntrica. De esta manera, los músculos y ligamentos podrían sufrir un estiramiento y posible disfunción temporomandibular.

También puede haber mordidas cruzadas posteriores uni o bilaterales, que podrían provocar desviaciones mandibulares y que acompañadas por una sobrecarga, las fuerzas colaterales podrían causar, igualmente, disfunción temporomandibular.

\section{Interferencia en protrusiva}

Las interferencias en protrusiva aparecen cuando la mandíbula avanza en protrusión y habitualmente se localizan en las vertientes mesiales de las superficies oclusales de los molares posteriores mandibulares y las superficies distales de los molares posteriores maxilares.

Se piensa que una interferencia de un molar en protrusión es, posiblemente, el tipo de interferencia más dañino que hay y sucede, habitualmente, en extrusiones y espacios sin dientes. El espacio dejado por una extracción dental facilita que el molar remanente distal pueda sufrir un desplazamiento o una inclinación hacia delante (Fig.8), o bien una extrusión del antagonista que interfiere el movimiento mandibular de protrusión (Fig. 9). También puede aparecer con los cordales, en malposiciones dentarias y en mordidas cruzadas.

La interferencia en protrusiva establece un área de fulcro en la mandíbula que puede provocar una subluxación condilar en el lado afecto. El paciente desvía la mandíbula en distintas direcciones con el fin de evitar esta interferencia. Pero esta desviación también se acompaña de un estiramiento y torsión de los ligamentos y tejidos blandos que afectan a todo el sistema articular.

\section{Interferencia en trabajo}

No son tan lesivas como las interferencias en lado de balanceo, pero pueden, también, en determinados casos desarrollar un estiramiento de los ligamentos y músculos así como un desplazamiento condilar anómalo en la articulación ya que puede rotar sobre el eje del lado afecto.

Las interferencias en el lado de trabajo tienen, también, influencia en la aparición de microtraumatismos y desgaste dentinario en ese mismo lado debido a las fuerzas oclusales horizontales ejercidas.

Se localiza, habitualmente en oclusiones de tipo I, entre las vertientes linguales de las cúspides vestibulares de los molares maxilares y las vertientes vestibulares de las cúspides bucales de los molares inferiores (Fig.10). 


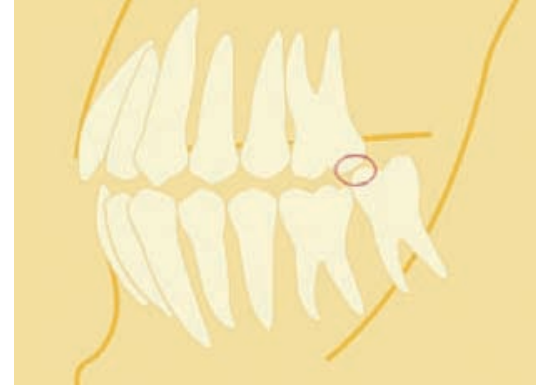

Figura 9

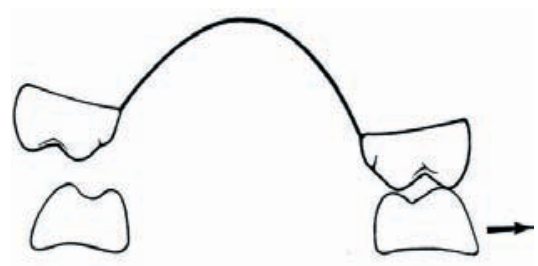

Figura 10

\section{Interferencia en balanceo}

En el lado de balanceo, o no trabajo, el cóndilo orbita o se traslada. La localización más frecuente de las interferencias en este lado es en las vertientes vestibulares de las cúspides linguales de los molares superiores y las vertientes linguales de las cúspides vestibulares de los molares mandibulares (Fig.12). Diferentes estudios demuestran la íntima relación que existe entre este tipo de interferencias y los TTM y en nuestra práctica clínica observamos, en la mayoría de las ocasiones, una mejoría que puede ir de moderada a muy evidente cuando se suprimen este tipo de contactos dentales en pacientes aquejados de dolor temporomandibular. En los últimos años, los trabajos que demuestran la nociva influencia de las interferencias en la articulación han ampliado su campo de estudio al aspecto postural mostrando que determinados grupos musculares relacionados con la ATM también pueden verse afectados de manera evidente influ-

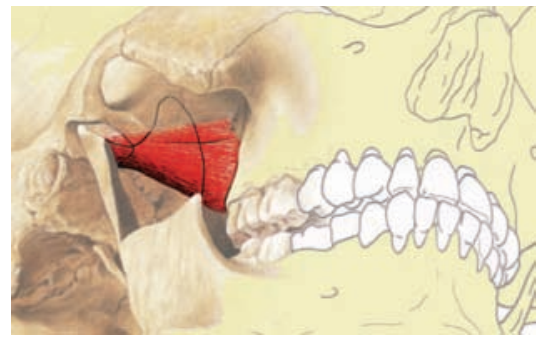

Figura 11. El pterigoideo lateral es un músculo especialmente sensible en pacientes con interferencias oclusales que debe explorarse siempre de manera funcional debido a su dificultad en la palpación.

yendo de manera indirecta en la articulación a modo de dolor miofascial heterotopico ${ }^{8,9}$. Cabe destacar que las interferencias en céntrica pueden coincidir con las de balanceo y ello tiene implicación en el ulterior tratamiento con un tallado selectivo.

Desde el punto de vista fisiopatológico, se produce un movimiento condilar anómalo con estiramiento y afectación de los tejidos blandos (Fig.11) del lado de no trabajo y desviación de la mandíbula que puede traumatizar la articulación.

Los vectores de fuerza son modificados y el fulcro en el lado de trabajo que representaba el bolo alimenticio se acompaña, ahora, por un segundo fulcro en el lado de balanceo, representado por la interferencia, lo que hace que el brazo de palanca sea mas corto, alterando de esta manera todo el sistema propioceptivo y neuromuscular del aparato estomatognático (Fig. 12).

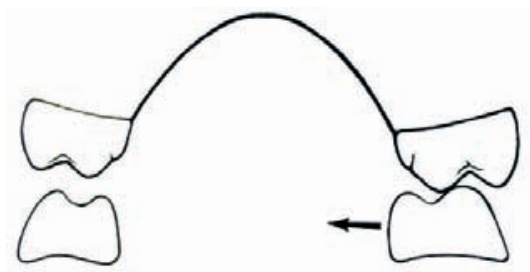

Figura $12 \mathrm{~A}$

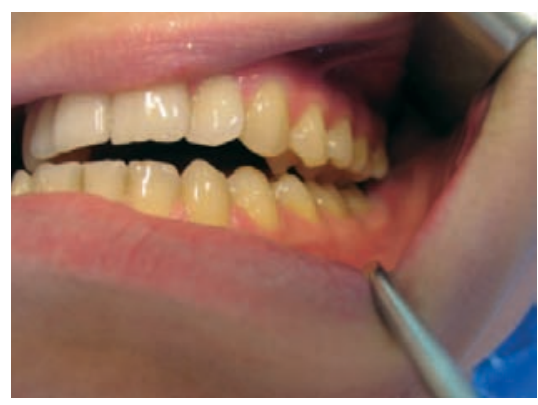

Figura 12B

\section{Interferencia en oclusión céntrica}

como veremos en el apartado siguiente, la coincidencia estructural y espacial entre la posición músculo esquelética estable y la oclusión céntrica o máxima intercuspidación, es la situación deseable y óptima en la dinámica articular. Sin embargo, independientemente de que esta relación sea la más idónea, puede suceder que, en ocasiones, el trayecto de la mandíbula hacia la oclusión céntrica se vea interrumpida por una interferencia que impida el correcto recorrido del cóndilo hacia la fosa. Ocurre, fundamentalmente, en malposiciones dentarias derivadas de espacios sin dientes (Fig.13), malformaciones esqueléticas o apiñamientos dentales. La repercusión clínica implica un microtraumatismo continuo cuya gravedad dependerá del grado de interferencia y la respuesta de los tejidos articulares.

\section{Discrepancia OC-RC}

La situación biomecánica idónea se cumpliría cuando la máxima intercus- 

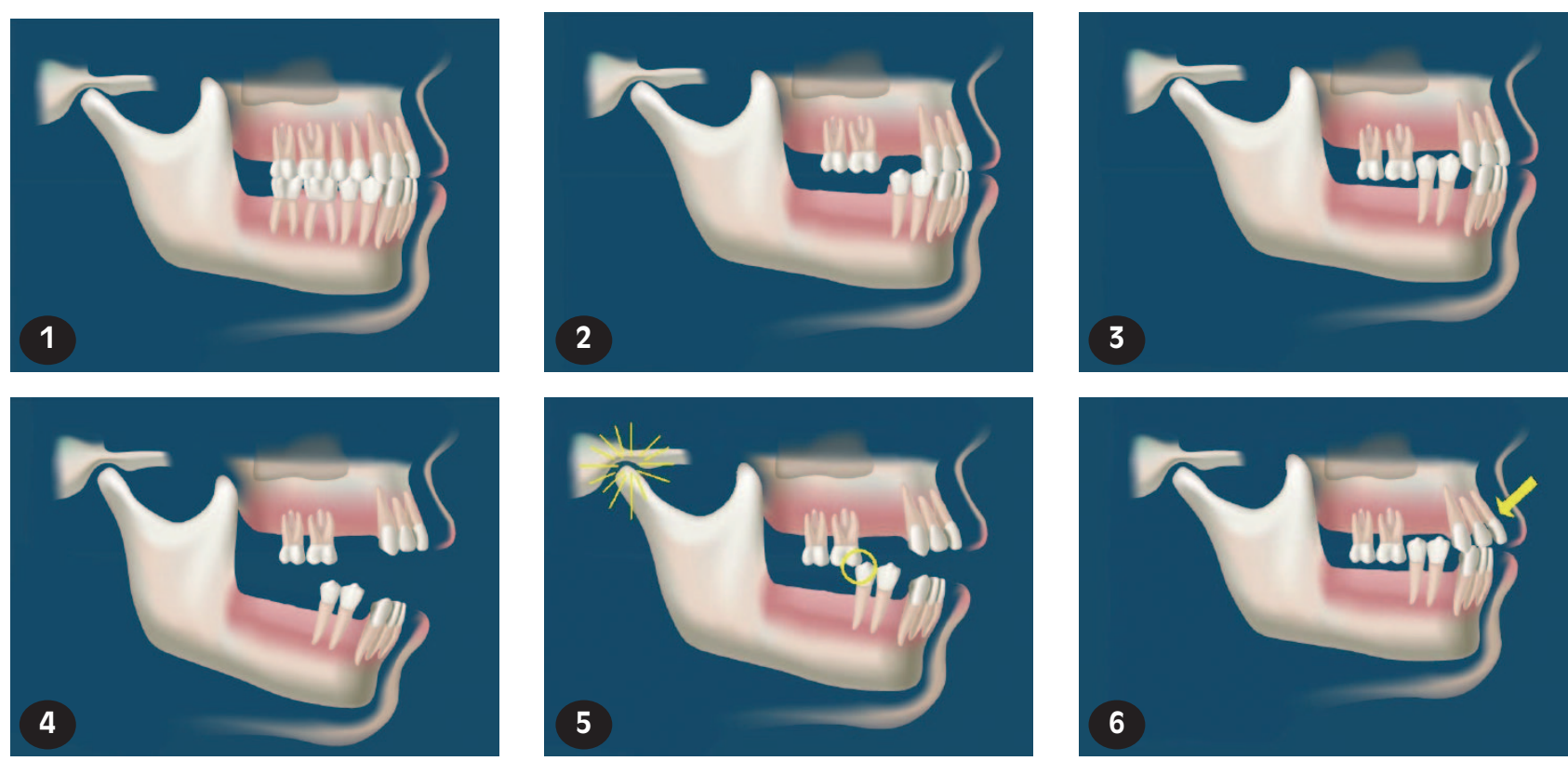

Figura 13. El desdentamiento parcial bimaxilar puede derivar en extrusiones y migraciones dentales. En ocasiones, el resultado es una malposición dentaria que puede interferir en la dinámica condilar alterando el trayecto hacia la cavidad glenoidea 1. Situación previa. 2. Edentulismo. 3. Extrusión y migración. 4. Apertura. 5. Cierre mandibular y desviación del trayecto normal por interferencia. 6. Splaying complementario por hipercontacto anterior.

Cortesía de Oris Educo.

pidación y en estabilidad ortopédica coincide con la posición articular musculoesquelética más estable ${ }^{10}$ (Fig.14). Sin embargo, puede existir un exceso del rango de movimiento mandibular desde esta posición de relación céntrica* a la posición de oclusión céntrica o máxima intercuspidación (Fig.15) que puede llegar hasta los $5 \mathrm{~mm}$.

Igualmente, un individuo puede aparentar una oclusión perfecta, en

\footnotetext{
* El concepto estático de relación céntrica hä sufrido a lo largo de los años una serie de modificaciones conceptuales basadas más en la biomecánica y fisiología articular, empleándose hoy en día términos más funcionales como estabilidad articular ortopédica, posición musculoesquelética estable, posición articular funcional óptima, etc. En cualquier caso sería la posición óptima de homeostasia articular en la que se valoran no sólo las superficies articulares sino también la función y los tejidos adyacentes.
}

clase I y sin alteraciones aparentes pero, una vez montados los modelos de estudio en relación céntrica, se puede apreciar una gran discrepancia con la máxima intercuspidación (Fig.16). En esta posición de relación céntrica puede aparecer una mordida abierta anterior ó unos contactos molares cúspide-cúspide y la aparición de patología dependerá fundamentalmente de dos elementos: la cohabitación con otros factores coadyudantes y la medida de la discrepancia entre las dos posiciones.

\section{Disminución de la dimensión vertical}

La reducción de la altura de corona clínica, la pérdida de soporte posterior debido a la ausencia (Fig.17), a la rotación 0 al desplazamiento de los molares (Fig.18), o también en pacientes portadores de prótesis extraible que con el paso del tiempo sufren una reabsorción ósea ${ }^{11}$ son distintas situaciones que derivan en una disminución de la dimensión vertical. La alteración de esta dimensión vertical puede repercutir en el estado neuromuscular, propioceptivo y postural ${ }^{12}$ quedando a expensas de la capacidad de adaptación de cada individuo, lo que reportará una gran variabilidad de respuesta. Por otra parte, la modificación de los vectores de fuerza craneales puede derivar en una intrusión y compresión condilar en el espacio retrodiscal, ricamente inervado y vascularizado, y ofrecer una explicación al componente doloroso en algunos de estos pacientes. Esta presión intraarticular en la ATM puede ser significativamente modificada después del 


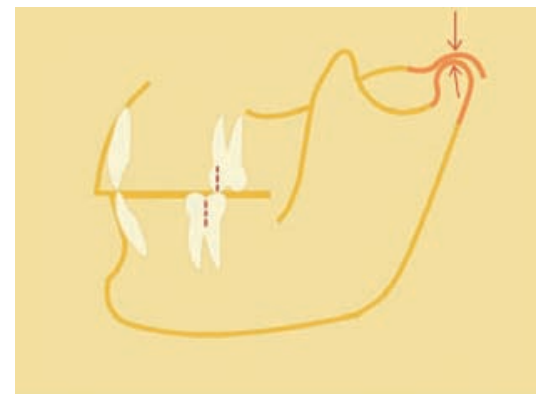

Figura 14

aumento de la dimensión vertical ${ }^{13}$ y reducirse de manera evidente el dolor retrodiscal.

La reacción neuronal propioceptiva proporcionada por el periodonto y los ligamentos está disminuida para actuar como mecanismo inhibitorio durante la contracción de los músculos elevadores y el cierre mandibular. El resultado es muy perjudicial pudiendo llegar, después de largos procesos traumáticos, a la perforación discal.

Como daño colateral, los dientes maxilares anteriores pueden actuar como stop oclusal en el cierre mandibular. Si esta situación se perpetúa en el tiempo, el periodonto cede a las fuerzas oclusales y los dientes anteriores podrían sufrir una migración anterior como los estudiadas en la fig. 5 .

\section{Aumento de la dimensión vertical}

Es una situación que se produce, generalmente, de manera iatrogénica y a partir de prótesis con una oclusión muy alta que obliga al paciente a mantener una posición de semiapertura y una separación permanente de las superficies articulares. Otra situación que puede desencadenar esta patología es el mal uso y abuso de placas o férulas oclusales.

El proceso histológico iatrogénico desencadenado parece ser una remo-

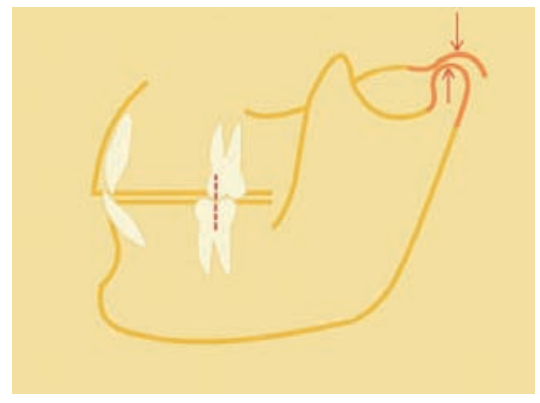

Figura 15

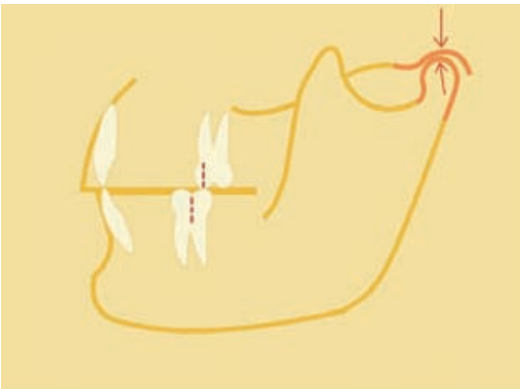

Figura 17

delación de las superficies articulares con aumento del volumen cartilaginoso y la formación de una estructura precondroblástica de mesenquima indiferenciado como mecanismo de compensación para preservar la íntima relación entre las superficies que quedarían alteradas por el aumento de espacio interarticular ${ }^{14}$. Igualmente, puede repercutir en el estado neuromuscular y propioceptivo que se manifiesta por la variación en la actividad electromiográfica .

\section{Alteraciones funcionales y morfoló- gicas}

En diversas revisiones bibliográficas ya se ha puesto de relieve la importancia de la oclusión como factor contribuyente en los trastornos temporomandibulares e incluso se ha valorado la opción de recuperar la función temporomandibular con tratamiento protésico y ortodóncico. Pero existen

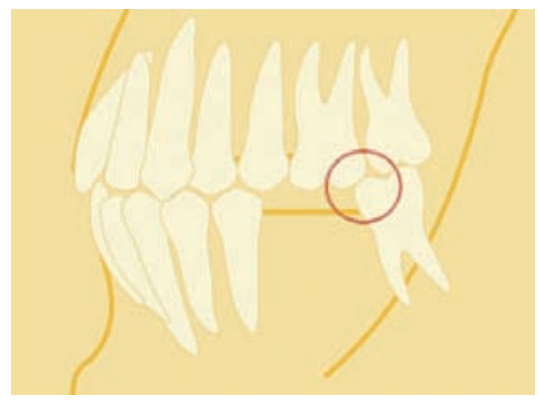

Figura 16

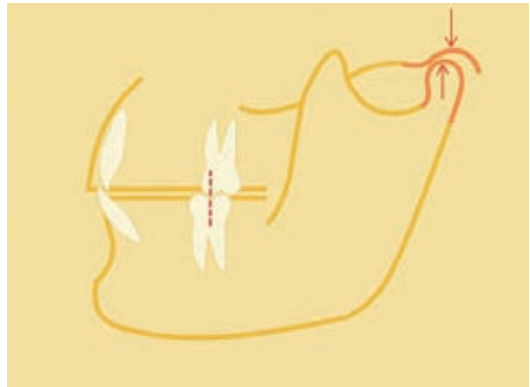

Figura 18

también otros componentes relacionados con la oclusión como son la masticación unilateral, la curva de spee, los espacios edéntulos, la postura, etc. que también pueden intervenir como cofactores contribuyentes en la presencia de TTM. Así, parece que hay una correlación muy evidente en pacientes con sintomatología temporomandibular y el lado donde mastican de manera exclusiva por lo que se pone de manifiesto la conveniencia de evitar esta práctica ${ }^{16}$. También existen distintos estudios que relacionan la curva de Spee como otro elemento más de disarmonía oclusal relacionado con los trastornos temporomandibulares. Entre los pacientes que presentan disfunción, la mayoría (69\%) presentan alteraciones en la curva de Spee, mientras que entre los que no la tienen, la mayoría (64\%) presentan una convexidad normal. Estas distribuciones estadísticas 
caracterizan a las alteraciones en la convexidad de la curva como un franco riesgo de disfunción ${ }^{17}$. Existe, también, una relación cuantitativa entre el número de dientes antagonistas con una posición de maloclusión y su mayor o menor implicación en la aparición de signos y síntomas en el sistema articular. También existen estudios bibliograficos que amplian la relación de los TTM con los estados de maloclusión dental a las alteraciones posturales ${ }^{18,19}$. Cabe destacar, por ejemplo, como la disposición de los espacios sin dientes en la cavidad bucal determinan no sólo su influencia en la ATM sino a nivel cefálico y postura ${ }^{20}$.

Los estudios, en conclusión, confirman una estrecha relación entre los factores oclusales y las alteraciones temporomandibulares ${ }^{21,22}$ y posturales y valoran de manera especial el diagnóstico y estudio oclusal individualizado de cada paciente para considerar y ponderar el correcto tratamiento.

\section{Opciones terapéuticas}

El arsenal terapéutico para abordar los trastornos cráneo-mandibulares es múltiple y variado y es, precisamente, el análisis etiopatológico el que determina en última instancia la opción terapéutica adecuada para cada individuo.

La norma básica y el punto de partida en el tratamiento de los TTM es optar, siempre que podamos, por la solución menos cruenta y reversible de las que dispongamos. Ya hemos estudiado previamente cómo una disarmonía oclusal o una alteración en la relación oclusión-articulación puede afectar de manera directa en la salud articular. Los métodos y las vías de corrección oclusal para solventar este mecanismo patológico de alteración articular se pueden resumir en cuatro: la rehabilitación protésica, el tallado selectivo, la corrección ortodóncica y la feruloterapia.

Las cuatro alternativas pueden desencadenar, en última instancia y de manera iatrogénica, un daño mayor e irreversible. Esto podría explicar, en parte, un cierto rechazo o reparo en la aplicación de estas opciones terapéuticas. Sin embargo, nosotros creemos que el éxito de la corrección oclusal radica en la indicación de la propia técnica y su adecuada praxis.

Observamos de manera reiterada como pacientes con una clara anomalía oclusal son tratados con fármacos, férulas, electroterapia, ultrasonidos, bio miofeed-back, infiltraciones, fisioterapia y otra suerte de tratamientos que, si bien es cierto que son reversibles y poco cruentos, suponen una terapia paliativa y sintomática que no modifican, en absoluto, la fuente real del trastorno, además de suponer, en ocasiones, un incremento del costo al paciente.

Como el propósito de este trabajo no es el estudio del tratamiento oclusal sino la demostración de que en ocasiones la corrección oclusal está irremediablemente relacionado con el éxito terapéutico en determinados tipos de pacientes, vamos a relatar de manera resumida cuales serían las posibilidades terapéuticas en la terapia oclusal según la clasificación de la tabla II: a) las maloclusiones son intervenidas, valoradas y tratadas por un ortodoncista, b)las interferencias deben ser suprimidas con un tallado selectivo por el odontólogo de manera protocolizada con un análisis clínico y oclusal mediante montaje previo en articulador semiajustable y c)las alteraciones en la dimensión vertical por un prostodoncista.

Los tratamientos con placas oclusales o férulas, nosotros creemos que deben reservarse, en principio, para los pacientes refractarios a las terapias anteriormente descritas y, en ocasiones, sólo como método de diagnóstico diferencial.

\section{Conclusiones}

La relación intrínseca entre alteración oclusal y trastorno articular no es un paradigma que se cumpla siempre. Sin embargo, el análisis oclusal debe ser siempre un requisito imprescindible en la exploración del paciente disfuncional ya que multitud de estudios a lo largo de los años han demostrado que puede representar un factor etiológico de primer orden y la corrección oclusal puede ser, en ocasiones, la opción terapéutica determinante en la resolución de un cuadro temporomandibular.

Un proceso terapéutico estandarizado y universal para todos los pacientes por igual, obtendrá, posiblemente, buenos resultados en muchas ocasiones, pero es absolutamente seguro que no podrán obtener buenos resultados en todos y cada uno de los casos que se presenten. La combinación y elección del método y técnica terapéutica, adecuado a cada caso y cada individuo en función del diagnóstico y la etiopatología, es la mejor vía para solventar de manera exitosa los distintos casos de trastornos cráneo mandibulares y evitar el elevado número de pacientes refrac- 
tarios al tratamiento. Adoptar esta actitud más racional implica una mayor preparación académica, un mayor estudio científico, una mayor dedicación al paciente y un equipo multidisciplinar para optar a la terapia idónea.

En particular, el tratamiento oclusal y el reestablecimiento de la homeostasia oclusal implica conocer la biomecánica particular de cada paciente y su correlación con sus parámetros oclusales y biológicos. Una interferencia oclusal puede ser absolutamente incapaz de provocar algún desequilibrio en un individuo y ser, sin embargo, muy dañina en otro paciente. Procesar esta variabilidad individual es el objetivo de la exploración del paciente disfuncional. Los factores coadyudantes y el entorno, la estabilidad funcional ó la posición estática y dinámica son diferentes entre los distintos casos por lo que discernir esta variabilidad individual es el objetivo de la exploración de nuestros pacientes.

\section{Bibliografla recomendada}

Para profundizar en la lectura de este tema, el/los autor/es considera/an interesantes los artículos que aparecen señalados del siguiente modo: ${ }^{*}$ de interés ${ }^{*}$ de especial interés.

1**. Oliveira W: Disfunções Temporomandibulares. Sao Paulo: Artes Médicas; 2002:135

2. Harper R, Misch E, Svenson K: Consideraciones funcionales y biológicas para la reconstrucción de la oclusión dentaria. Quintessence Int. 2000;4:34-35

3. Pullinger AG, Seligman DA, Jerolimoc V et al. A multiple logistic regression analysis of the risk and relative odds of temporomandibular disorders as a function of common occlusal features. J Dent Res. 1993;72:68-79.

4. Glickman I. Role of occlusion in etiology and treatment of periodontal disease. J Dent Res. 1991;50:119-204.

5. Ricketts R. Lecture given before occlusion faculty. University of Southern California School of Dentistry. 1993.

6. Stephens R, Lavigne G, Clark GT, Lund JP. Occlusal adjustment in periodontal therapy. Dental Association J. 1993;39:332-337.

7**. Geering A. Occlusal interference and functional disturbances of masticatory system. J Clinic Periodont. 1994;1:112-119.

Este estudio muestra que la oclusión puede intervenir de manera importante en pacientes con dolor facial y desglosa de manera pormenorizada los mecanismos implicados.

8**. Khono S. Exámen electromiográfico de las relaciones entre los episodios dolorosos del músculo esternocleidomastoideo y las interferencias oclusales. Quintessence.1992; 10:34-41

El ECM tiene la función de estabilizar la cabeza contra la función de la masticación y esto constituiría una explicación para el hecho de relacionar las alteraciones de la oclusión con el dolor en el ECM. A través del EMG se demuestra la actividad del músculo variando distintas posturas y distintas situaciones oclusales por medio de férulas que obligan al paciente a alterar su oclusión.

9. Leiva M. Effects of laterotrusive Occlusal Écheme and body position on bilateral sternocleidomastoid EMG activity. J Craniomandib Pract.2003;2:107-108

10. Pahkala R. Variación en la función del sistema masticatorio en 1008 niños rurales. J Clin Pediatr Dent. 1991;1:25-30.

11. Araki A, Yokoyarna T, Murakamu H, lto Y, Maeda H, Kameyama Y. Effect of decreased vertical occlusion on mandibular condyle of senescence-accelerated mouse. J Dent Res. 1999;3:78:194.

12. Moyers RE, Miralles R, Dodss C. Skeletal contributions to occlussal development. J Craniomandib Pract.2001;4:230.

13. Nitzan DW. Intraarticular pressure in the functioning hurnan temporomandibular joint and its alteration by uniform elevation of the occlusal plane. $\mathrm{J}$ Oral and Maxillofac Surg. 1994;52:67-680.

14. Rashed M Z, Sharawy M. Histopathological and inmunocytochemical studies of the effect of raised occlusal vertical dimension on the rabbit. J Craniomandib Pract. 1993:4:291-6.

15. Manss A, Miralles R. Influence of vertical dimension in the treatment of myofascial pain dysfunction síndrome. J Prosthetic Dent. 1983;5:700-709.

16. Reinhardt et al. The unilateral chewing phenomenon, occlusion and TMD. J Craniomandib Pract. 2006;3:167-169.

17. Machado M, Quintana S. Disfunción craneomandibular y sus relaciones con los factores morfológicos de la oclusión. Instituto superior de ciencias médicas de Villa Clara. Revista cubana de ortodoncia. 1995;3:56-59.

18*. Sharifi Milani R, Deville D. Relationship between dental occlusion and posture. J Craniomandib Pract. 2000;2:127.

Se utilizó el test de Fukuda y unos dispositivos interoclusales para demostrar que es posible modificar la actitud postural de los pacientes modificando la oclusión dental.

19. Mafosky H. The influence of forward head posture on dental occlusion. J Craniomandib Pract. 2000;1:30-31

20. Yoshino G. Changes in head position due to occlusal supporting zone loss during clenching. J Craniomandib Pract .2003;2:91

21. Celic R, Jerolimoc V. A study of the influence of occlusal factors and parafunction. Int $\mathrm{J}$ prosthodont. 2002; 15:43-48.

$22 *$. Abd Al Hadi L. Prevalence of temporomandibular disorders in relation to some occlusal parametres. J Prosthetic Dental. 2003;70:345-350.

Con este estudio se pone de manifiesto que existen distintos parámetros oclusales y cada uno de ellos puede influir de manera distinta en los trastornos temporomandibulares. 